\title{
MAQUILA Y MAQUILADORA
}

\section{MAQUILA \& MAQUILADORA}

\author{
Zelma Wong Torres* \\ Docente Principal de la Facultad de Ciencias Contables, UNMSM \\ Luisa Elena Salcedo Guzmán** \\ Docente Asociada de la Facultad de Ciencias Contables, UNMSM
}

[Recepción: Agosto de 2010 / Conformidad: Setiembre de 2010]

\author{
"El hombre es un fin en sí mismo y no un medio \\ $o$ instrumento al servicio de otros hombres". \\ "Solo las cosas tienen precio, \\ los hombres no tienen precio \\ porque tienen dignidad". \\ Emmanuel Kant
}

\section{RESUMEN}

La maquila y maquiladora se debe abordar como un problema que los profesionales debemos conocer y participar ampliamente para ayudar a los más pobres a salir de ese nivel, porque las empresas bajo este sistema se están apropiando en forma sistemática de los países emergentes, bajo la justificación de que no se tiene educación, salud, ni trabajo, pues consideran que la migaja que nos dan es más que suficiente para todos nosotros.

Es necesario que los profesionales peruanos miremos a nuestro país como el poderoso que es, que no supo hasta ahora abrir los ojos y reconocer las riquezas que posee, aquí es necesario recordar la expresión de don Antonio Raymondi "Perú es un mendigo sentado en un banco de oro" que hoy en día recién está despertando y como se está descubriendo, los empresarios apuestan que no debemos despertar; no debemos dejarlos porque entonces hermano peruano, com-

\begin{abstract}
The maquila and maquiladora must be addressed as a problem that professionals must understand and participate extensively to help the poor rise above that level, because firms under this system are systematically appropriating emerging countries, justified that do not have education, health, no job, considering the pittance they give us is more than enough for all of us.

It is necessary for Peruvian professionals look to our country as powerful it is, who failed so far to open their eyes and recognize the wealth they have, here is to remember the words of Don Antonio Raimondi "Peru is a beggar sitting on a bench of gold today is just like waking up and being discovered, entrepreneurs are betting that we should not awaken, we must not let them because then Peruvian brother, understand that the wealthy businessman takes advantage of our ignorance and our poverty to be useful fools
\end{abstract}

1 Magíster en Ciencias Contables, UNMSM; Contadora Pública Colegiada Certificada. Docente Investigadora de la Facultad de Ciencias Contables, UNMSM. E-mail: zwongt@hotmail.com

2 Contadora Pública Colegiada. Docente Investigadora de la Facultad de Ciencias Contables, UNMSM.E-mail: luisalcedag@ yahoo.es 
prenderás que el rico empresario se aprovecha de nuestra ignorancia y nuestra pobreza por ser tontos útiles que no sabemos defender y explotar lo que tenemos, en nuestro propio beneficio, tanto hoy como a futuro para nuestras generaciones venideras.

Debemoslos profesionales demostrar nuestra real competencia para que ya no nos exploten como siempre lo han hecho los grandes empresarios, debemos exigir el $50 \%$ de todas sus ganancias que sean repartidas con los trabajadores, la solución la tenemos, formemos empresa uniéndonos, aportando nuestra opiniones e ideas, que analizadas y expuestas se mejoren entre nosotros mismos.

Palabras clave: Maquila y maquiladora, sistema social y productivo, cohesión social.

\section{INTRODUCCIÓN}

Podemos empezar este artículo sobre pobreza y como en nuestro país estos famosos sistemas, según ellos novedosos, sin nosotros percibirlo, para lo cual meditamos sobre El Manifiesto de la doctrina social de la iglesia: "el desarrollo nunca estará plenamente garantizado por fuerzas que en gran medida son automáticas e impersonales, ya provengan de las leyes de mercado o de políticas de carácter internacional", sostiene Benedicto XVI.

Debemos ver los efectos perniciosos sobre la economía real de una actividad financiera mal utilizada:

- Los flujos migratorios no realizados adecuadamente.

- La explotacion de los recursos de la tierra, sin reglas.

- Aumento exagerado de las desigualdades.

- Aumento de la desigualdad entre grupos sociales dentro del país.

- Tipo de subdesarrollo derrochador y consumista frente a situaciones persistentes de miseria deshumanizadora.

- Erosión de la cohesion social e impacto negativo en lo económico por las diferencias sociales. do not know how to defend and exploit what we have in our own benefit, both now and in the future for our generations to come.

We must show our real professional competence that we no longer operating as they have always been big business, we require $50 \%$ of all profits to be distributed to workers, the solution we have formed joint uniting and make our opinions and ideas that will improve analyzed and displayed among ourselves.

Key words: The maquila and maquiladora, Social and productive system, social cohesion and impact.

- El hombre abusa de los instrumentos económicos que han tiranizado la libertad de las personas y organizaciones sociales, aumentando la pobreza relativa.

La economía de mercado debe ser justicia distributiva tendiendo a la justicia social, el mercado no debe regirse al valor de los bienes pues no hay cohesión social para su funcionamiento.

Sin solidaridad y confianza el mercado no puede cumplir adecuadamente su función económica.

La gestión de la empresa no debe ser únicamente el interés de los propietarios, sino en beneficio de todos los sujetos que contribuyen a su existencia como ente: propietario, trabajadores, clientes y proveedores, no a la especulación; se debe proponer a la búsqueda de beneficio para todos, no se debe explotar sin aportar a la sociedad para el logro de un sólido sistema productivo y social. La Encíclica tiene por objeto procurar el desarrollo humano integral.

En el Perú, lacerante e inhumana falta de alusión a la primera infancia periodo vital del ser humano, $46 \%$ de niños menores de 5 años del área rural padecen de desnutrición y el 29\% de desnutrición crónica, los menores 
de 3 años son los menos atendidos en salud, de los menores de 36 meses a escala nacional, $57 \%$ padecen de anemia y esta es la edad en que se forman las capacidades individuales, cognitivas y productivas del menor. Algún futuro es posible con esta realidad? Para ello es necesario desprenderse de los apetitos personales y realizar un pacto entre los políticos, para invertir en la primera infancia, hoy, ajena en la agenda de los políticos pues estos no votan, no protestan, no hacen huelga, no toman carreteras. El Estado, la empresa y la sociedad deben invertir en la infancia, factor clave para erradicar la pobreza y avizorar un desarrollo para el país, en el futuro. Subsiste un círculo vicioso de la pobreza, la cual como herencia pasa de padres a hijos y de hijos a hijos y a nietos.

Según Bernardo Klinksberg ${ }^{3}$ al invertirse en educación y salud el crecimiento e investigación científica y tecnológica dará un crecimiento económico, el mismo que debe ir acompañado de inversiones importantes en la población, una inversión a largo plazo que dará un capital humano de clase A.

Inversión en América Latina:

\section{Salud}

América Latina 3.5\% del PBI

Países desarrollados 6\% del PBI

\section{Educación}

América latina 4\% del PBI

Israel, Japón, Corea $8 \%$ del PBI

Perú 3\% del PBI

Sin embargo, en el Perú se suprime asignaturas, tales como, educación psicomotriz que representan las matemáticas y la educación por el arte que es la comunicación integral.

\section{MAQUILA Y MAQUILADORA}

Maquila "es la porción de lo molido, que corresponde al molinero"; así nació la palabra y viene del árabe hispano 'maquila' que quiere decir medida y este tiene sus raíces en el árabe clásico maquilad que significa "cosa medida”. De todo esto se derivó el verbo 'maquilar' que se comenzó a usar por los año 1734 y 'maquiladora' en el siglo XX. En la edad media se usaba para designar la porción de grano, harina o aceite que se le pagaba al molinero por su trabajo de moler dichos productos, por cuenta y riesgo del que pagaba la comisión fija por el trabajo realizado.

Maquiladora se comienza a usar en el siglo XX como una forma de producción industrial empleado en la producción de otros bienes, por este hecho no cambia de propiedad sino que se convierte en un servicio a la empresa, se utiliza en el proceso productivo de enviar a otra diferente para una actividad de maquila; o sea hacer una producción por cuenta ajena introduciéndose la terminología al léxico económico por su etimología.

Se usó mucho por el empresario para aprovecharse de las míseras condiciones laborales existentes, en principio no tienen que ser de explotados, porque así se originó, pagándose salarios inferiores a los reales.

La maquiladora es la zona de procesamiento para la exportación siendo una planta manufacturera de propiedad local o extranjera.

En América Latina aparece en los años 1960 y 1970 bajo el auspicio Estados Unidos, para contener la migración; en los años 90 toma un gran impulso la liberación del comercio internacional y la mundialización de la economía, llamada globalización, a partir de 1990 buscando las empresas bajar al máxi-

1 Economista argentino experto mundial de lucha contra la pobreza asesor principal del Programa de Naciones Unidas para el Desarrollo 2009. 
mo sus costos de producción especialmente en mano de obra pagando muchas veces 1.5 dólares americanos por hora mientras que en Estados Unidos 17.2 o sea 11 veces más.

Este es un modelo empresarial de exportación, por el que una empresa se compromete a producir un artículo para un tercero, quien comercializa el producto generalmente con su marca propia, se utiliza insumos y tecnología importados solo utilizan la mano de obra local y lo exportan. Los insumos intermedios empleados en la producción de otros bienes no cambian de propietario regresando a su lugar de origen.

Los países industrializados para mejorar su competitividad internacional aprovechan los salarios bajos de los países menos desarrollados y en muchos de los casos la mano de obra es femenina. El fondo de estas maquiladoras es que no transfieren tecnología avanzada a los países donde funcionan, pues se dedican a ensamblar insumos importados desde otros países para reexportarlos, gozando de exenciones tributarias ya que se ubican en zonas especiales de exportación en donde se benefician de numerosas ventajas que le ofrecen los países receptores, las maquilas no promueven el desarrollo de las regiones en las que se instalan porque precisamente aprovechan las condiciones de subdesarrollo de la región que ofrece mano de obra barata.

Los estudiosos asocian a las maquiladoras como precariedad, falta delibertad, nolibertad de asociación de negocios, salarios de hambre, largas y agotadoras jornadas de trabajo.

En las transacciones internacionales creadas por las fronteras políticas y aduaneras; maquila se usa cuando ambas unidades no están relacionadas desde el punto de vista de la propiedad, en cambio cuando el producto se traslada de un país a otro para un segmento de la producción esto es conocido con el nombre de maquila aunque ambas pertenez- can a la misma empresa siendo este el privilegio del comercio internaciones.

En América Latina estas actividades económicas nacionales o extranjeras ejecutan una parte muy precisa en el proceso de producción. Se encuentran ubicadas en zonas francas o zonas procesadoras de exportación y obtienen ventajas de exoneración de impuestos y todas las facilidades que le ofrecen los países para su instalación. Ellos prefieren ubicarse en zonas francas fronterizas, ideal zonas estratégicas las fronteras de los países, siendo México quien más experiencia tiene de esta explotación.

Las empresas que instalan una industria maquiladora, reciben del país: Infraestructura adecuada, servicio de agua, energía eléctrica, puertos, telecomunicaciones, sistemas viales, legislación laboral flexible, facilidades económicas.

Los países en vía de desarrollo tienen que cumplir con estos requisitos para que el empresario se ubique en su país.

Por otro lado, una industria maquiladora de exportación instalada en un país debería ofrecer:

- Promover empleo en zonas con problemas demográficos.

- Obtener ingresos de divisas para fortalecer la balanza de pagos.

- Establecer industrias y capacitación solo en actividades primarias no industriales.

- Obtener mercado para productos nacionales e incrementar los ingresos en las zonas marginadas económicamente.

- Proceso de aprendizaje industrial que busque erradicar la deficiente calidad de mano de obra.

- Inversiones en infraestructura industrial

- Impulsar el desarrollo de la franja fronteriza

- Generación de tecnologías automatizadas. 
- Fortalecimiento de la competitividad en el país huésped.

\section{Características de la producción en la maquila}

- Baja inversión per cápita

- Bajos salarios, existiendo en muchos casos el sistema remunerativo al destajo.

- Inadecuadas condiciones sociales y laborales.

- Supresión de sindicatos.

- Largas jornadas laborales, en promedio 10 horas diarias, en muchos casos con horas extras obligatorias.

- Estructura de la fuerza laboral constituida en su mayoría por mujeres y menores de 18 años.

- La empresa tiene su propio sistema de regulación de conflictos.

- Alta rotación de personal.

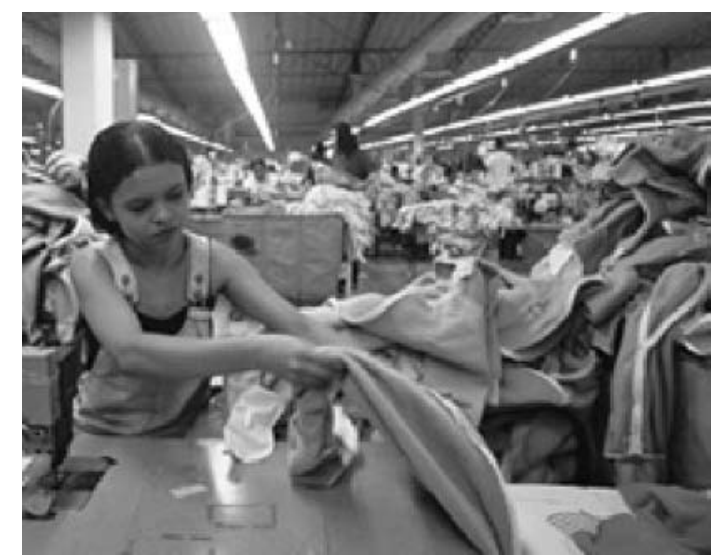

\section{CÓMO AFECTA LA MAQUILA EN LA ECONOMÍA DE CADA PAÍS}

Las maquilas generan competencias entre países pobres para atraer capitales, también entre regiones del mismo país.

Implementan cambios en la legislación laboral del comercio exterior teniendo un tratamiento especial al capital extranjero
Manejo de las condiciones ambientales, recursos naturales y la biodiversidad.

\section{GUATEMALA}

Para lograr mayores niveles de ganancia se aumentó la jornada laboral a 12 horas diarias y el uso de estimulantes para elevar el rendimiento, administrándose a mujeres embarazadas, provocándose nacimiento de niños deformes según denuncia de la Unión Sindical de Trabajadores de Guatemala (UNSINTRAGUA).

\section{ARGENTINA}

Con Carlos Saúl Menen se implementaron varias leyes laborales para favorecer a empresas proporcionando un puesto de trabajo costara una tercera parte menos que antes, pero se crearon nuevos puestos de trabajo, más bien, se prefirió pagar horas extras.

\section{PERÚ}

La flexibilización de leyes laborales permitió despidos masivos de mineros impedidos de formar sindicatos, los empresarios se han negado a atender los reclamos de los trabajadores.

\section{MAQUILA EN PERÚ}

Los empresarios nacionales de la maquila, invierten generalmente en infraestructura y administración de los parques industriales; mientras que la empresa extranjera que se sirve de esta, invierte en tecnología y administración de la fabricación de sus productos dentro de estos parques industriales. 
En nuestro país se encuentra operando la llamada Zona Franca, ubicada en Tacna; (ZOFRATACNA), además de tres Centros de Exportación, Transformación, Industria, Comercialización y Servicios (CETICOS), ubicados en Paita, Ilo y Mollendo. Y desde el 2006 la Zona Económica Especial de Puno (ZEEDEPUNO) creado con la finalidad de contribuir al desarrollo económico de Puno.

Todas estas zonas y centros brindan el servicio de maquila.

ZOFRATACNA: Su principal actividad consiste en depositar y custodiar mercancía procedente del exterior para su posterior comercialización, interno o externo.

Se permite el siguiente tratamiento tributario:

- Exoneración de Impuesto a la Renta para mercancías que se exportan.

- Exoneración del IGV a los insumos.

- Exoneración del ISC a los insumos.

- Suspensión de todo impuesto a las maquinarias.

- Exoneración del impuesto Advalorem.

CETICOS: Centros administrados por al CONAFRAN. Entre las actividades que desarrollan estos centros se encuentra la maquila o ensamblaje, almacenamiento de mercancía proveniente del exterior o del territorio nacional para su posterior exportación y actividades de servicios como embalaje, rotulado, clasificación, entre otros.

Las mercancías nacionales o nacionalizadas que ingresan a los CETICOS para efecto de maquila, no pueden ser nacionalizadas nuevamente, sino que deben ser transformados o utilizados en la actividad desarrollada o exportada.

ZEEDEPUNO: Entre las actividades que puede desarrollarse en esta zona especial se encuentran: Maquila, ensamblaje, actividades industriales y agroindustriales, servicio de almacenamiento o distribución, embalaje, desembalaje, envasado, clasificación, entre otros.
En cuanto a los aspectos tributarios, los usuarios que realicen actividades en ZEEDEPUNO, están exonerados de: Impuesto a la Renta, IGV, ISC, impuesto de promoción municipal y todo tributo, sean estos del gobierno central, regional o municipal.

\section{MAQUILA Y RÉGIMEN}

En el caso de la maquila se da el Régimen de Tráfico de Perfeccionamiento de Activos (TPA), estos a su vez son dos:

\section{Régimen de administración temporal}

Permite al productor nacional requerir insumos extranjeros, el ingreso al Perú de materias primas, productos intermedios y envases no pagan derechos de importación siempre que sean incorporados a productos de exportación dentro de los próximos 24 meses, incluye exención de tasa, contribución e impuestos exigibles a la importación.

\section{Régimen de reposición de mercancía en franquicia}

Exoneración automática de los derechos arancelarios e impuestos que gravan la importación de material o mercancías que una vez nacionalizados han sido transformados o agregados a productos de exportación, definitivamente pueden ser destinadas al mercado interior o a la producción para la exportación; en este caso los exportadores gozaran del beneficio de exoneración de impuestos.

\section{CONCLUSIONES}

- La maquila es un sistema de subcontratación de servicios. Para aprender está bien, pero una vez que se aprende debe independizarse para no ser explotado. Se 
usa mucho en México, Chile, Perú y otros países, y es sobre la fabricación de un producto determinado en todo su proceso, esto lo puede hacer un emprendedor o microempresario que quiere aprender, luego independizarse del maquilador que, en buen romance, es un explotador, porque al contar con capital fuerte, evade mediante este sistema las diferentes obligaciones laborales y sociales que tendría que cumplir con esos trabajadores.

- Maquila es un modelo que busca el uso de tecnología con una fuerza laboral oprimida.

- Este modelo de maquilación, ha adquiridola característica de enclave, ya que la inversión extranjera persigue beneficiarse explotando recursos humanos y recursos naturales.

- La maquilación, se da en zonas donde las empresas se pueden establecer pagando un costo mínimo de impuestos y donde la explotación de niños y mujeres es muy alta, si es que nuestros compatriotas se dejan los salarios son de hambre a ella pertenecen pequeñas empresas locales las cuales subcontratadas por corporaciones multinacionales

- Maquiladora son centros de trabajo cuya actividad se concentra en el ensamblaje, transformación y/o reparación de componentes destinados a la exportación. Como condición necesaria y suficiente para su operación; gozan de un régimen fiscal de excepción lo cual les permite importar insumos sin pagar aranceles y exportar pagando solamente un arancel que fue agregado en México, otras de sus ventajas es aprovecharse de las ventajas competitivas, mano de obra barata, mayormente femenina.

- Debemos ver los efectos perniciosos sobre la economía real de una actividad financiera mal utilizada, los flujos migratorios no realizados adecuadamente, la explotación sin reglas de los recursos de la tierra. Aumento exagerado de las desigualdades. Aumento de la desigualdad entre grupos sociales dentro del país. Tipo de subdesarrollo derrochador y consumista frente a situaciones persistentes de miseria y deshumanización.

Las maquiladoras sacrifican ingresos fiscales para el gobierno del país huésped.

\section{REFERENCIAS BIBLIOGRÁFICAS}

1. Bendiski, I., Gordinez, V. Mendoza, M. La industria maquladora. Revista Trayectoria. Año 4, N.o 7.2002.

2. Buitelaar, R., Vandisck, P. Exportacionesd e manufacturas: Desafíos para las pequeñas economías de América Latina. Revista Cepal N.o 6. Abril 1997.

3. Fernández L. Ispanoteca-Lengua y cultura foro de Maquila consultas y archivo.

4. Fernández, R. El espejismo de las maquilas. http://rcci.net/globalizacion/2003/ fg353.htm

5. Klinksberg, B. Economista argentino experto mundial de lucha contra la pobreza. Asesor del Programa de las Naciones Unidas para el Desarrollo, 2009.

6. Moncada, M. Maquilaje con empuje. www.ni.laprensa.ni/cronologico/2004/ marzo/10/economica/economia-2004 0310-02.html

7. Quinteros, C. Por qué el sector maquilador necesita mejorar en el cumplimiento de los derechos laborales. Revista Online 2004: http://saenzpe.intra.gov.ar/Prointal/cap5.htm

8. Romero, J. La industria maquiladora y el sector confección venezolano. Trabajo de ascenso. FCES. Universidad del $\mathrm{Zu}$ lia. Venezuela, 2001.

9. Wolf, M., Berna, L, Martinez, C. Dinámica 2003 en la maquila de Centroamérica. Economía. FUNDAS-PAD. Plataforma Sindical Común Centro América PSCC. 2003. 